\title{
CONTINUOUS FUNCTIONS ON COMPACT SPACES WITHOUT PERFECT SUBSETS
}

\author{
WALTER RUDIN
}

I. Introduction. Let $\mathfrak{e}(X)$ be the set of all continuous complexvalued functions on a compact Hausdorff space $X$, made into a Banach algebra by defining $\|f\|=\sup |f(x)|(x \in X)$. A subset $\mathcal{F}$ of $\mathfrak{C}(X)$ is said to be separating if to every pair of points $p, q \in X$ there is a function $f \in \mathcal{F}$ for which $f(p) \neq f(q)$.

If some subset of $X$ is homeomorphic to the Cantor set, then $\mathfrak{e}(X)$ contains proper subalgebras which are closed and separating but which are not maximal ideals; indeed, there are maximal proper subalgebras of $\mathfrak{e}(X)$ with these properties [3]. Recently, Civin and Yood [1] have studied regular Banach algebras with a countable set of maximal ideals. Their work contains the following result: if $X$ is countable and if $a$ is a maximal closed proper subalgebra of $\mathfrak{e}(X)$ which is separating, then $Q$ is a maximal ideal of $\mathfrak{e}(X)$.

In the present paper we shall study $\mathfrak{e}(Q)$, where $Q$ is a compact Hausdorff space without perfect subsets (we adopt the convention that the empty set is not perfect); thus every nonempty closed subset $F$ of $Q$ contains a point which is not a limit point of $F$. There are uncountable spaces $Q$; for instance, the one-point compactification of any discrete space, or the set of all ordinals which do not exceed a given ordinal, with the usual interval topology. It is easily seen that $Q$ is totally disconnected, and that the isolated points of $Q$ form a dense subset of $Q$.

The principal result about $\mathfrak{e}(Q)$ is that every closed subalgebra of $\mathfrak{e}(Q)$ is self-adjoint. This implies that the maximal ideals of $\mathfrak{e}(Q)$ are the only closed separating proper subalgebras of $\mathfrak{e}(Q)$, a fact previously unnoticed even in the countable case. The linear functionals on the Banach space $\mathfrak{C}(Q)$ (i.e., the regular Borel measures on $Q$ ) are also shown to have a very simple structure.

Throughout this paper the letter $Q$ will stand for a compact Hausdorff space without perfect subsets.

\section{The continuous functions on $Q$.}

THEOREM 1. If there exists a continuous mapping $f$ of $Q$ onto a compact Hausdorff space $X$, then $X$ contains no perfect subset.

Presented to the Society, August 24, 1956; received by the editors March 18, 1956. 
Proof. Suppose $X$ contains a perfect set $P$. There exists a compact subset $E$ of $Q$, minimal with respect to the property that $f(E)=P$. Our hypothesis about $Q$ implies that $E$ has an isolated point $q$; put $E^{\prime}=E-\{q\}$. Then $E^{\prime}$ is compact, the minimality of $E$ shows that $f\left(E^{\prime}\right)$ is a proper subset of $P$, and the compactness of $f\left(E^{\prime}\right)$ implies that $f(q)$ is not a limit point of $f\left(E^{\prime}\right)$, hence not a limit point of $P$. This contradicts the assumption that $P$ is perfect, and the theorem follows.

Since every uncountable compact subset of the plane contains a perfect subset, we obtain the following corollary:

Theorem 2. If $f \in \mathfrak{e}(Q)$, then $f(Q)$ is countable.

TheOREм 3. Every closed subalgebra $a$ of $\mathfrak{C}(Q)$ is self-adjoint.

Proof. Choose $f \in a$. Theorem 2 shows that $f(Q)$ is a countable compact subset of the complex plane. Let $E$ be the union of $f(Q)$ and the origin. Since $E$ is a compact set without interior points which does not separate the plane, there exists, for each positive integer $n$, a polynomial $P_{n}$ such that $P_{n}(0)=0$ and $\left|P_{n}(z)-\bar{z}\right|<1 / n$ on $E[2]$. If $f_{n}(q)=P_{n}(f(q))$ for $q \in Q$, then $f_{n} \in Q$ and $\left\|f_{n}-\bar{f}\right\|<1 / n$, so that $Q$ contains $\bar{f}$, the complex conjugate of $f$.

THEOREM 4. If $a$ is a closed separating subalgebra of $\mathfrak{e}(Q)$ and $a$ is not a maximal ideal of $\mathfrak{e}(Q)$, then $Q=\mathfrak{e}(Q)$.

Proof. This is an immediate consequence of Theorem 3 and the Stone-Weierstrass theorem.

III. The regular Borel measures on $Q$. There is a decreasing family of subspaces of $Q$, obtained in the following manner. Put $Q_{0}=Q$, let $\alpha$ be an ordinal, and suppose that compact spaces $Q_{\beta}$ have been defined for all $\beta<\alpha$. If $\alpha$ is not a limit ordinal, then $\alpha=\beta+1$ for some $\beta$, and we let $Q_{\alpha}$ be the set of all limit points of $Q_{\beta}$. If $\alpha$ is a limit ordinal, we put $Q_{\alpha}=\bigcap_{\beta<\alpha} Q_{\beta}$.

The absence of perfect sets in $Q$ shows that $Q_{\alpha+1}$ is a proper subset of $Q_{\alpha}$ (if $Q_{\alpha}$ is not empty), so that there exists a first ordinal, say $\gamma$, such that $Q_{\gamma}$ is empty. If $\gamma$ were a limit ordinal, then $Q_{\gamma}$ would be the intersection of a family of nonempty compact sets with the finite intersection property, so that $Q_{\gamma}$ could not be empty. It follows that there is a last ordinal $\lambda$ such that $Q_{\lambda}$ is not empty. Consequently, $Q_{\lambda}$ is a finite space.

By a regular Borel measure on $Q$ we mean a non-negative, finite, countably additive set function $m$ defined for all Borel sets in $Q$, such that $m(E)=\sup m(F)=\inf m(G)$, where $F$ runs through all 
compact subsets of $E$, and $G$ runs through all open supersets of $E$.

THEOREM 5. If $m$ is a regular Borel measure on $Q$ and if $m(E)=0$ for every set $E$ which consists of a single point, then $m(Q)=0$ (i.e., $m$ vanishes identically).

Proof. Suppose $m(Q)>0$. Since $Q_{\lambda}$ is finite, $m\left(Q_{\lambda}\right)=0$, and there is a first ordinal, say $\alpha$, such that $m\left(Q_{\alpha}\right)<m(Q)$. If $\alpha=\beta+1$, then $m\left(Q_{\beta}\right)=m\left(Q_{\alpha}\right)+m\left(Q_{\beta}-Q_{\alpha}\right)$. But $Q_{\beta}-Q_{\alpha}$ contains no infinite compact set, hence $m\left(Q_{\beta}-Q_{\alpha}\right)=0$, and $m\left(Q_{\beta}\right)=m\left(Q_{\alpha}\right)$, contradicting our choice of $\alpha$.

Hence $\alpha$ is a limit ordinal. For any open set $G$ which contains $Q_{\alpha}$ there exists then some $\beta<\alpha$ such that $Q_{\beta} \subset G$. Since $\beta<\alpha, m\left(Q_{\beta}\right)$ $=m(Q)$. Hence $m(G)=m(Q)$ for every open set $G$ containing $Q_{\alpha}$. The regularity of $m$ shows then that $m\left(Q_{\alpha}\right)=m(Q)$. This contradiction proves the theorem.

The Riesz representation theorem, together with Theorem 5, now yields the following description of the linear functionals on $\mathfrak{e}(Q)$ :

THEOREM 6. If $T$ is a bounded linear functional on the Banach space $\mathfrak{e}(Q)$, then there exist points $q_{n} \in Q$ and complex numbers $c_{n}$ such that $\sum\left|c_{n}\right|<\infty$ and

$$
T f=\sum_{n=1}^{\infty} c_{n} f\left(q_{n}\right)
$$

As an application of this, we give a short proof of a theorem which, for countable $Q$, is due to Civin and Yood [1]. We preface the theorem with the following remark, which is easily proved: for every $q \in Q$ there is a unique ordinal $\alpha(q) \leqq \lambda$ such that $q$ is an isolated point of $Q_{\alpha(q)}$, but $q$ is a limit point of $Q_{\beta}$ if $\beta<\alpha(q)$.

Theorem 7. Let $\&$ be a closed linear subspace of $\mathfrak{e}(Q)$ which, for each $q \in Q$, contains a function $f_{q}$ with the following properties: $f_{q}(q)=1$, $f_{q}(p)=0$ if $p \in Q_{\alpha(q)}-\{q\}$. Then $\mathfrak{L}=\mathfrak{e}(Q)$.

Proof. If $\mathscr{L} \neq \mathfrak{C}(Q)$, there is a functional $T$, given by Theorem 6 , which annihilates $\mathscr{L}$ but is not identically zero. Let $\alpha$ be the smallest of the ordinals $\alpha\left(q_{n}\right)$, where we consider only those values of $n$ for which $c_{n} \neq 0$. Then $\alpha=\alpha\left(q_{i}\right)$ for some $i$, and $T f_{q_{i}}=c_{i}$. Since $T$ annihilates $\mathscr{L}, c_{i}=0$, a contradiction.

\section{REFERENCES}

1. Paul Civin and Bertram Yood, Regular Banach algebras with a countable space of regular maximal ideals, Proc. Amer. Math. Soc. vol. 7 (1956) pp. 1005-1010. 
2. S. N. Mergelyan, On the representation of functions by series of polynomials on closed sets, Doklady Akademii Nauk SSSR. (N.S.) vol. 78 (1951) pp. 405-408; Amer. Math. Soc. Translations, no. 85, Providence, 1953.

3. Walter Rudin, Subalgebras of spaces of continuous functions, Proc. Amer. Math. Soc. vol. 7 (1956) pp. 825-830.

UNIVERSITY OF ROCHESTER

\section{A SCHWARZ INEQUALITY FOR CONVEX OPERATOR FUNCTIONS}

\section{CHANDLER DAVIS}

For any Hilbert space $\mathfrak{K}$, let $\mathscr{K}$ be the totality of bounded selfadjoint operators with spectrum contained in an interval $I$, which need not be finite. If $f$ is a function from $\mathfrak{K}$ to the self-adjoint operators on $\mathfrak{F C}$ obtained from a bounded real-valued function $f_{0}$ on $I$ by the spectral theorem (that is, by $f(A)=\int_{-\infty}^{\infty} f_{0}(\lambda) d E_{\lambda}$, where $E_{\lambda}$ is the spectral resolution of $A$ ), then $f$ is called an operator function (associated with $I$ ). An operator function $f$ is defined, once $f_{0}$ is given, for all such $\Re$. An operator function $f$ associated with $I$ is convex provided $A, B$ in $\mathcal{K}$ and $0 \leqq t \leqq 1$ imply

$$
f(t A+(1-t) B) \leqq t f(A)+(1-t) f(B) .
$$

THEOREM. The operator function $f$ associated with $I$ is convex if and only if

$$
\text { (A) for } A \in \mathfrak{K} \text { and for any projection } P, \quad P f(P A P) P \leqq P f(A) P \text {. }
$$

Seymour Sherman suggested to me the problem of characterizing operator functions $f$ satisfying (A) by conditions on the corresponding real-valued functions $f_{0}$. (He remarked that the square is such a function $^{1}$ but the absolute value is not. Sherman has also made an attack on the present theorem by other methods.) The characterization is provided by combining the present theorem with characterizations of convex operator functions [1]. In particular, it is necessary but far from sufficient that $f_{0}$ be convex and analytic.

Proof. From the definition follow these useful facts about any operator function:

Presented to the Society, October 27, 1956; received by the editors March 21, 1956.

$1\left((\mathrm{PAP})^{2} x, x\right)=(P A P x, A P x) \leqq(A P x, A P x)=\left(P A^{2} P x, x\right)$. 